\title{
Projection-Based Local and Global Lipschitz Moduli of the Optimal Value in Linear Programming
}

\author{
M.J. Cánovas ${ }^{1} \cdot$ M.J. Gisbert ${ }^{2}$ D. Klatte ${ }^{3}$ J. Parra ${ }^{1}$ (1) \\ Received: 11 May 2021 / Accepted: 14 September 2021 / Published online: 17 October 2021 \\ (c) The Author(s) 2021
}

\begin{abstract}
In this paper, we use a geometrical approach to sharpen a lower bound given in [5] for the Lipschitz modulus of the optimal value of (finite) linear programs under tilt perturbations of the objective function. The key geometrical idea comes from orthogonally projecting general balls on linear subspaces. Our new lower bound provides a computable expression for the exact modulus (as far as it only depends on the nominal data) in two important cases: when the feasible set has extreme points and when we deal with the Euclidean norm. In these two cases, we are able to compute or estimate the global Lipschitz modulus of the optimal value function in different perturbations frameworks.
\end{abstract}

Keywords Lipschitz modulus · Optimal value · Orthogonal projections · Linear programming $\cdot$ Variational analysis

Dedicated to Franco Giannessi on the occasion of his 85th birthday.

Communicated by Boris S. Mordukhovich.

This research has been partially supported by Grants PGC2018-097960-B-C21 and PID2020-116694GB-I00 from MICINN, Spain, and ERDF, ‘A way to make Europe,' European Union.

$\bowtie \quad$ J. Parra

parra@umh.es

M.J. Cánovas

canovas@umh.es

M.J. Gisbert

mgisbert@est-econ.uc3m.es

D. Klatte

diethard.klatte@business.uzh.ch

1 Center of Operations Research (CIO), Miguel Hernández University of Elche, Elche, Spain

2 Department of Statistics, Universidad Carlos III de Madrid, Getafe, Spain

3 Institut für Betriebswirtschaftslehre, Universität Zürich, Zürich, Switzerland 
Mathematics Subject Classification 90C31 - 49J53 - 49K40 - 90C05 15 A60

\section{Introduction}

This paper exploits geometrical aspects of balls (associated with arbitrary norms) in order to sharpen a lower bound, given in [5, Theorem 4.1], on the Lipschitz modulus of the optimal value of linear programs under canonical perturbations, i.e., tilt perturbations of the objective function together with right-hand side (RHS) perturbations of the inequality system defining the constraints.

Lipschitz properties of optimal values and optimal solutions of perturbed linear programs have been systematically studied since the 1970s and 1980s. In particular, it is well known that the optimal value function, when restricted to solvable problems (i.e., with finite optimal value), is Lipschitzian on bounded subsets of the parameter space, see, e.g., $[12,17]$. The latter also follows from results of parametric linear optimization (see, e.g., [10, Chapter 8] or [1, Chapter 5.5]), saying that the parameter space of solvable problems is a convex polyhedral cone and the restricted optimal value function is continuous and piecewise quadratic there. Moreover, for a fixed objective function (respectively, for a fixed RHS) the corresponding 'partial' optimal value function is piecewise linear and convex (respectively, concave) on its effective domain (which is a convex polyhedral set), hence globally Lipschitzian there, see, e.g., [10, Chapter 6], [1, Chapter 5.5] or [16,17]. For recent detailed discussions of the history of Lipschitz analysis in linear optimization, we refer to the papers $[4,5]$.

Clearly, to have an exact expression of the Lipschitz modulus of the optimal value function at a (given) nominal solvable problem is a matter of interest in the sensitivity analysis. Such an exact expression is obtained in [5, Theorem 5.2] in the case when the nominal set of optimal solutions is bounded, and moreover, this expression is given exclusively in terms of the nominal problem's data (parameters and solutions), not involving problems in a neighborhood; in this sense, we often use the term pointbased expression. The reader is addressed to [5] for further comments and references and $[3,8,9,14]$ and references therein for a wider view on Lipschitz moduli and related variational concepts.

As an immediate antecedent to the current analysis, [5, Theorem 4.1] provides a point-based upper bound on the Lipschitz modulus of the optimal value under tilt perturbations of the objective function (for a fixed RHS) and [5, Theorem 3.1] gives an exact expression for RHS perturbations and fixed objective. The present paper completes the picture about the Lipschitzian behavior of the optimal value function by providing:

- A lower bound on the Lipschitz modulus for fixed RHS (and perturbable objective), which equals the exact modulus in the cases when either the nominal feasible set contains no lines or the norm considered in the space of decision variables $\left(\mathbb{R}^{n}\right)$ is the Euclidean norm. Note that the nominal feasible set contains no lines if and only if it has extreme points, which obviously includes the case when it is bounded.

- The exact global Lipschitz modulus for fixed RHS in the two cases mentioned above. 
- The exact global Lipschitz modulus for fixed objective (in all cases).

- An upper bound on the global Lipschitz modulus for canonical perturbations under certain assumptions ensuring the modulus' finiteness.

The main ingredient in this study is the operator norm of the orthogonal projection mapping on the (nonzero) linear subspace spanned by the left-hand side (LHS) coefficients of the constraint system with respect to the usual inner product in $\mathbb{R}^{n}$. Such a subspace is nothing else but the orthogonal one to the lineality space (see [13]) of any nonempty feasible set. Observe that, since we are not perturbing the LHS of the constraints, this lineality space is fixed. The two special cases mentioned above (the existence of extreme feasible points or the use of the Euclidean norm) make the referred operator norm equal 1, independently of the values of the LHS coefficients.

The structure of the paper is as follows: Section 2 introduces the necessary notation and preliminary results. Section 3 provides some ad hoc results on dual norms of projections. Then, Section 4 computes the referred local and global moduli for partial perturbations (with either the objective function or the RHS being fixed). The case of canonical perturbations is tackled in Section 5. We finish the paper with a section of conclusions.

\section{Notation and Preliminaries}

Given a subset $X \subset \mathbb{R}^{n}$, by $\operatorname{conv} X$, cone $X$, and $\operatorname{span} X$ we denote the convex hull, the conical convex hull, and the linear subspace spanned by $X$. It is assumed that cone $X$ and $\operatorname{span} X$ always contain $0_{n}$, the zero vector of $\mathbb{R}^{n}$. In particular, cone $\{\varnothing\}=\operatorname{span}\{\emptyset\}=$ $\left\{0_{n}\right\}$. The orthogonal subspace to $X$ is denoted as $X^{\perp}:=\left\{y \in \mathbb{R}^{n}: y^{\prime} x=0\right.$ for all $x \in X$ \}. Let us also denote by $\operatorname{extr} X$ the set of extreme points of a convex set $X \subset \mathbb{R}^{n}$.

From the topological side, int $X$ and $\operatorname{bd} X$ denote, respectively, the interior and the boundary of a given subset $X$ of any topological space.

Throughout the paper, we are concerned with the parameterized linear program

$$
\pi: \operatorname{Inf} c^{\prime} x \text { subject to } \bar{a}_{t}^{\prime} x \leq b_{t}, t \in T=\{1, \ldots, m\}
$$

where $x \in \mathbb{R}^{n}$ is the decision variable, regarded as a column vector, i.e., $\mathbb{R}^{n} \equiv \mathbb{R}^{n \times 1}$, the LHS coefficient vector $\bar{a}_{t} \in \mathbb{R}^{n}$ is given (fixed) for each $t \in T$, with some $\bar{a}_{t}$ being nonzero, the prime stands for transposition, and $\pi \equiv(c, b) \in \mathbb{R}^{n} \times \mathbb{R}^{T}$, with $b=\left(b_{t}\right)_{t \in T}$, being the parameter to be perturbed, (for simplicity, hereafter we write $\pi=(c, b))$ either locally, around a given nominal value $\bar{\pi}=(\bar{c}, \bar{b})$, or globally.

Associated with our parametric family (1) we consider the feasible set mapping, $\mathcal{F}: \mathbb{R}^{T} \rightrightarrows \mathbb{R}^{n}$, the optimal value function, $\vartheta: \mathbb{R}^{n} \times \mathbb{R}^{T} \longrightarrow[-\infty,+\infty]$, and the optimal set mapping, $\mathcal{F}^{o p}: \mathbb{R}^{n} \times \mathbb{R}^{T} \rightrightarrows \mathbb{R}^{n}$, given, respectively, by

$$
\begin{aligned}
\mathcal{F}(b) & :=\left\{x \in \mathbb{R}^{n}: \bar{a}_{t}^{\prime} x \leq b_{t}, t \in T\right\}, \\
\vartheta(\pi) & :=\inf \left\{c^{\prime} x: x \in \mathcal{F}(b)\right\}, \\
\mathcal{F}^{o p}(\pi) & :=\left\{x \in \mathcal{F}(b): c^{\prime} x=\vartheta(\pi)\right\},
\end{aligned}
$$


under the convention inf $\varnothing:=+\infty$.

We consider $\mathbb{R}^{n}$ endowed with an arbitrary norm $\|\cdot\|$, whereas the parameter spaces $\mathbb{R}^{T}$ and $\mathbb{R}^{n} \times \mathbb{R}^{T}$ are, respectively, equipped with the norms given by

$$
\|b\|_{\infty}:=\max _{t \in T}\left|b_{t}\right| \quad \text { and } \quad\|(c, b)\|:=\max \left\{\|c\|_{*},\|b\|_{\infty}\right\}
$$

with $\|c\|_{*}:=\max _{\|x\|=1}\left|c^{\prime} x\right|$. Hereafter $B, B_{*}$ and $B_{2}$ stand for the closed unit balls associated with $\|\cdot\|,\|\cdot\|_{*}$ and the Euclidean norm, $\|\cdot\|_{2}$, in $\mathbb{R}^{n}$.

Given $\bar{\pi}=(\bar{c}, \bar{b}) \in \operatorname{dom} \mathcal{F}^{o p}:=\left\{\pi=(c, b) \in \mathbb{R}^{n} \times \mathbb{R}^{T}: \mathcal{F}^{o p}(c, b) \neq \emptyset\right\}$ (the effective domain of $\mathcal{F}^{o p}$ ), the (local) Lipschitz modulus of $\vartheta$ at $\bar{\pi}$ is defined as

$$
\operatorname{lip} \vartheta(\bar{\pi}):=\limsup _{\pi, \tilde{\pi} \rightarrow \bar{\pi}, \pi \neq \tilde{\pi}} \frac{|\vartheta(\pi)-\vartheta(\tilde{\pi})|}{\|\pi-\tilde{\pi}\|},
$$

(under the convention $(+\infty)-(+\infty)=(-\infty)-(-\infty)=0)$. Then, lip $\vartheta(\bar{\pi})$ equals $+\infty$ when $\bar{\pi} \in$ bd $\operatorname{dom} \mathcal{F}^{o p}$. We can avoid infinite values of the Lipschitz modulus by restricting $\vartheta$ to dom $\mathcal{F}^{o p}$ (which in finite linear programming coincides with the set of bounded problems, i.e., having a finite optimal value). Following [5], we consider $\vartheta^{R}:=\left.\vartheta\right|_{\text {dom }} \mathcal{F} o p$. Obviously, $\operatorname{lip} \vartheta^{R}(\bar{\pi})=\operatorname{lip} \vartheta(\bar{\pi})$ for $\bar{\pi} \in \operatorname{int} \operatorname{dom} \mathcal{F}^{o p}$, whereas [5, Theorem 5.2] shows that $\operatorname{lip} \vartheta^{R}(\bar{\pi})$ is always finite for $\bar{\pi} \in \operatorname{dom} \mathcal{F}^{o p}$.

Analogously, we can define the global Lipschitz modulus of $\vartheta^{R}$ as

$$
\operatorname{g-lip} \vartheta^{R}:=\sup _{\pi, \tilde{\pi} \in \operatorname{dom} \mathcal{F}^{o p}, \pi \neq \tilde{\pi}} \frac{|\vartheta(\pi)-\vartheta(\tilde{\pi})|}{\|\pi-\tilde{\pi}\|} .
$$

In this paper, we start by considering the cases of $c$-perturbations (tilt perturbations), where $\bar{b} \in \operatorname{dom} \mathcal{F}$ remains fixed, and $b$-perturbations (RHS perturbations), for a fixed

$$
\bar{c} \in C:=-\operatorname{cone}\left\{\bar{a}_{t}, t \in T\right\}
$$

in order to guarantee dual consistency. Observe that, as it is well known in ordinaryfinite-linear programming (LP), $(c, b) \in \operatorname{dom} \mathcal{F}^{o p}$ if and only if $b \in \operatorname{dom} \mathcal{F}$ and $c \in C$ (see, e.g., [2, Section 4.3]). In other words,

$$
\operatorname{dom} \mathcal{F}^{o p}=C \times \operatorname{dom} \mathcal{F}
$$

Specifically, given $\bar{\pi}=(\bar{c}, \bar{b}) \in \operatorname{dom} \mathcal{F}^{o p}$, we consider the partial optimal value functions $\vartheta_{\bar{b}}^{R}: C \longrightarrow \mathbb{R}$ and $\vartheta_{\bar{c}}^{R}: \operatorname{dom} \mathcal{F} \longrightarrow \mathbb{R}$ given by

$$
\vartheta_{\bar{b}}^{R}(c):=\vartheta(c, \bar{b}) \text { and } \vartheta_{\bar{c}}^{R}(b):=\vartheta(\bar{c}, b) \text { for }(c, b) \in \operatorname{dom} \mathcal{F}^{o p} \text {. }
$$

Some results of the paper appeal to the condition $\bar{c} \in \operatorname{int} C$, provided that $\bar{\pi}=(\bar{c}, \bar{b}) \in$ $\operatorname{dom} \mathcal{F}^{o p}$, which is known to be equivalent to the boundedness of $\mathcal{F}^{o p}(\bar{\pi})$ (see, e.g., [6, Corollary 9.3.1]). 
As it has been advanced in the introduction, a key role in our analysis is played by the orthogonal projection mapping, denoted by $P$, on $\operatorname{span}\left\{\bar{a}_{t}, t \in T\right\}$ with respect to the usual inner product in $\mathbb{R}^{n}$. More in detail, the exact modulus $\operatorname{lip} \vartheta_{\bar{b}}(\bar{c})$ is obtained when

$$
\|P\|_{*}:=\max _{\|u\|_{*}=1}\|P u\|_{*}=1
$$

Example 3 shows that condition $\|P\|_{*}=1$ is not superfluous. Observe that always $\|P\|_{*} \geq 1$.

As in [5, formula (7)], we consider the set (which goes back to [4, Section 2.2])

$$
\mathcal{E}^{o p}(\pi):=\operatorname{extr}\left(\mathcal{F}^{o p}(\pi) \cap \operatorname{span}\left\{\bar{a}_{t}, t \in T\right\}\right), \pi \in \operatorname{dom} \mathcal{F}^{o p}
$$

For completeness, let us observe that whenever $\pi \in \operatorname{dom} \mathcal{F}^{o p}, \mathcal{E}^{o p}(\pi)$ is a nonempty and finite set. Indeed, we can write

$$
\begin{aligned}
& \mathcal{F}^{o p}(\pi) \cap \operatorname{span}\left\{\bar{a}_{t}, t \in T\right\} \\
& \quad=\left\{x \in \mathbb{R}^{n} \mid \bar{a}_{t}^{\prime} x \leq b_{t}, t \in T, c^{\prime} x=\vartheta(\pi), Q x=0\right\},
\end{aligned}
$$

where $Q$ is the matrix whose rows form a basis of $\left\{\bar{a}_{t}, t \in T\right\}^{\perp}$. Then, the nonemptiness and finiteness of set of extreme points of the previous set can be deduced, for instance, from [2, Theorems 2.2 and 2.3.].

For simplicity in the notation, for our nominal problem $\bar{\pi}=(\bar{c}, \bar{b})$, we denote as $e\left(\mathcal{E}^{o p}(\bar{\pi}), 0_{n}\right)$ the Hausdorff excess of $\mathcal{E}^{o p}(\bar{\pi})$ over $\left\{0_{n}\right\}$. In other words,

$$
e\left(\mathcal{E}^{o p}(\bar{\pi}), 0_{n}\right):=\max _{x \in \mathcal{E}^{o p}(\bar{\pi})}\|x\|
$$

Firstly, we are going to consider the case of $c$-perturbations, where $\bar{b} \in \operatorname{dom} \mathcal{F}$ remains fixed. The next result can be seen as the starting point of this paper:

Theorem 1 (see [5, Theorem 4.1]) Let $\bar{\pi} \in \operatorname{dom} \mathcal{F}^{o p}$. Then,

$$
d\left(0_{n}, \mathcal{F}^{o p}(\bar{\pi})\right) \leq \operatorname{lip} \vartheta_{\bar{b}}^{R}(\bar{c}) \leq e\left(\mathcal{E}^{o p}(\bar{\pi}), 0_{n}\right) .
$$

Moreover, if we assume that $\bar{c} \in \operatorname{int} C$, then

$$
\operatorname{lip} \vartheta_{\bar{b}}^{R}(\bar{c})=e\left(\mathcal{F}^{o p}(\bar{\pi}), 0_{n}\right)
$$

Remark 1 When $\bar{c} \in \operatorname{int} C$ (equivalently, $\mathcal{F}^{o p}(\bar{\pi})$ is bounded), [5, Theorem 4.1] also shows that $e\left(\mathcal{F}^{o p}(\bar{\pi}), 0_{n}\right)$ equals the calmness modulus of $\vartheta_{\bar{b}}^{R}$ at $\bar{c}$. See [4] for further details on the calmness modulus of $\vartheta^{R}$ in the different perturbation settings.

Remark 2 Throughout the paper, the quantities $d\left(0_{n}, \mathcal{F}^{o p}(\bar{\pi})\right)$ and $e\left(\mathcal{E}^{o p}(\bar{\pi}), 0_{n}\right)$ frequently appear. At this moment, let us write some comments about the computation of these two scalars. Paper [7] is focussed on the computation of 
the minimum norm solution for an LP problem, in other words, to the computation of $d\left(0_{n}, \mathcal{F}^{o p}(\bar{\pi})\right)$, which is done by reformulating this problem as an unconstrained minimization problem with a convex and smooth objective function and applying a Newton-type method. As commented there, paper [7] (see also references therein) presents an alternative approach to the standard method based on the Tikhonov regularization ([15]). On the other hand, in the case when $\bar{c} \in \operatorname{int} C$, we have

$$
e\left(\mathcal{E}^{o p}(\bar{\pi}), 0_{n}\right)=e\left(\operatorname{extr} \mathcal{F}^{o p}(\bar{\pi}), 0_{n}\right)=e\left(\mathcal{F}^{o p}(\bar{\pi}), 0_{n}\right),
$$

where the last equality comes from the convexity of the norm. In this way, we can apply [11, Theorem 1.1] to derive an upper bound for $e\left(\mathcal{F}^{o p}(\bar{\pi}), 0_{n}\right)$ in terms of the problem's data. Despite the finiteness of $\mathcal{E}^{o p}(\bar{\pi})$, to the authors knowledge, the practical computation of $e\left(\mathcal{E}^{o p}(\bar{\pi}), 0_{n}\right)$ in the case when $\bar{c} \notin \operatorname{int} C$ remains as open problem.

The following example comes from [5, Example 4.2] (see more details therein) and provides a geometrical motivation for using projections in the present paper, as well as it shows that the second inequality in (5) can be strict. In this sense, it is worth mentioning that in [5, Example 4.1] the first inequality holds strictly. Recall that $B_{*}$ stands for the closed unit ball with respect to norm $\|\cdot\|_{*}$. See [13, Theorem 15.2] for a characterization of all possible norms in $\mathbb{R}^{n}$ in terms of their closed unit balls.

Example 1 [5, Example 4.2] Consider $\mathbb{R}^{2}$ endowed with the norm given by

$$
\|x\|:=\max \left\{\left|2 x_{1}+x_{2}\right|,\left|2 x_{1}+3 x_{2}\right|\right\},
$$

for which $B_{*}=\operatorname{conv}\left\{ \pm(2,1)^{\prime}, \pm(2,3)^{\prime}\right\}$. Consider the nominal problem in $\mathbb{R}^{2}$

$$
\begin{aligned}
\bar{\pi} & \text { minimize } x_{1} \\
& \text { subject to }-x_{1} \leq-2 .
\end{aligned}
$$

Then, $e\left(\mathcal{E}^{o p}(\bar{\pi}), 0_{2}\right)=\left\|(2,0)^{\prime}\right\|=4$ and $\operatorname{lip} \vartheta_{\bar{b}}^{R}(\bar{c})=2$. If we considered the Euclidean norm in $\mathbb{R}^{2}$, then both quantities would equal 2 for the same $\bar{\pi}$.

We finish this preliminary section with the counterpart of Theorem 1 for canonical perturbations.

Remark 3 A point-based expression of $\operatorname{lip} \vartheta_{\bar{c}}^{R}(\bar{b})$ is given in [5, Theorem 3.1]. The reader is addressed there for details. We omit it here in order to avoid additional notation and definitions.

Theorem 2 [5, Theorems 3.1 and 5.2 and Corollary 5.1] Let $\bar{\pi} \in \operatorname{dom} \mathcal{F}^{o p}$. Then,

$$
\operatorname{lip} \vartheta^{R}(\bar{\pi}) \leq \operatorname{lip} \vartheta_{\bar{c}}^{R}(\bar{b})+e\left(\mathcal{E}^{o p}(\bar{\pi}), 0_{n}\right) .
$$

If, additionally, $\bar{c} \in \operatorname{int} C$, then equality holds in (6), which reads as

$$
\begin{aligned}
\operatorname{lip} \vartheta^{R}(\bar{\pi}) & =\operatorname{lip} \vartheta_{\bar{c}}^{R}(\bar{b})+e\left(\mathcal{F}^{o p}(\bar{\pi}), 0_{n}\right) \\
& =\operatorname{lip} \vartheta_{\bar{c}}^{R}(\bar{b})+\operatorname{lip} \vartheta_{\bar{b}}^{R}(\bar{c}) .
\end{aligned}
$$




\section{Dual norms of orthogonal projections}

Let us denote by $\mathcal{H}$ the class of all linear subspaces of $\mathbb{R}^{n}$ not reduced to $0_{n}$ and, for each $H \in \mathcal{H}$, consider the orthogonal projection on $H, P_{H}: \mathbb{R}^{n} \longrightarrow H$, with respect to the usual inner product in $\mathbb{R}^{n}$. Evidently, $P_{H} u$ is the (unique) closest point to $u \in \mathbb{R}^{n}$ in $H$ with respect to the Euclidean norm in $\mathbb{R}^{n}$, but we focus on the norm of the linear mapping $P_{H}$, when $\mathbb{R}^{n}$ and $H$ are endowed with the dual norm $\|\cdot\|_{*}$ (recall that our 'primal' norm $\|\cdot\|$ is an arbitrary one). Let us write

$$
\left\|P_{H}\right\|_{*}=\max _{\|u\|_{*}=1}\left\|P_{H} u\right\|_{*} .
$$

Obviously $\left\|P_{H}\right\|_{*} \geq 1$ whenever $H \in \mathcal{H}$. We are particularly interested in the case $H=\operatorname{span}\left\{\bar{a}_{t}, t \in T\right\}$, whose associated projection we are denoting by just $P$. For instance, in Example 1 we have $\|P\|_{*}=2$ since $P\left(B_{*}\right)=[-2,2] \times\{0\}$ and $(1,0)^{\prime} \in$ bd $B_{*}$.

Next we present some technical lemmas. The first one appeals to the well-known fact that all norms in $\mathbb{R}^{n}$ are equivalent. Observe that $\left\|P_{H}\right\|_{2}=1$ whenever $H \in \mathcal{H}$.

Lemma 1 Let $0<\alpha \leq \beta$ be such that $\alpha\|\cdot\|_{2} \leq\|\cdot\|_{*} \leq \beta\|\cdot\|_{2}$ (or, equivalently, $\left.\beta^{-1} B_{2} \subset B_{*} \subset \alpha^{-1} B_{2}\right)$. Then,

$$
\sup _{H \in \mathcal{H}}\left\|P_{H}\right\|_{*} \leq \frac{\beta}{\alpha} .
$$

Proof For all $H \in \mathcal{H}$ and all $u \in B_{*}$, we have

$$
\left\|P_{H} u\right\|_{*} \leq \beta\left\|P_{H} u\right\|_{2} \leq \beta\|u\|_{2} \leq \frac{\beta}{\alpha}\|u\|_{*} .
$$

The following example shows that inequality (7) may be strict even in the case when $\alpha$ and $\beta$ are chosen sharp (the supremum and the infimum, respectively, of all possible ones).

Example 2 In the case of Example 1, we have the sharp $\alpha=1 / \sqrt{13}, \beta=\sqrt{2}$, and a routinary computation gives

$$
\max _{H \in \mathcal{H}}\left\|P_{H}\right\|_{*}=\frac{1+\sqrt{26}}{2} .
$$

More in detail, if we rotate (with respect to the origin) the extreme points of $B_{*}$ an angle $\theta \in[0, \pi]$, which is equivalent to rotate the horizontal axis an angle $-\theta$, we can check that the function

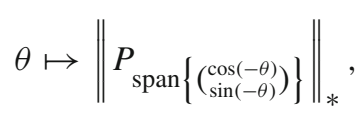

whose graph is given in Figure 1, attains its maximum at $\theta=\arctan (5+\sqrt{26})$. 


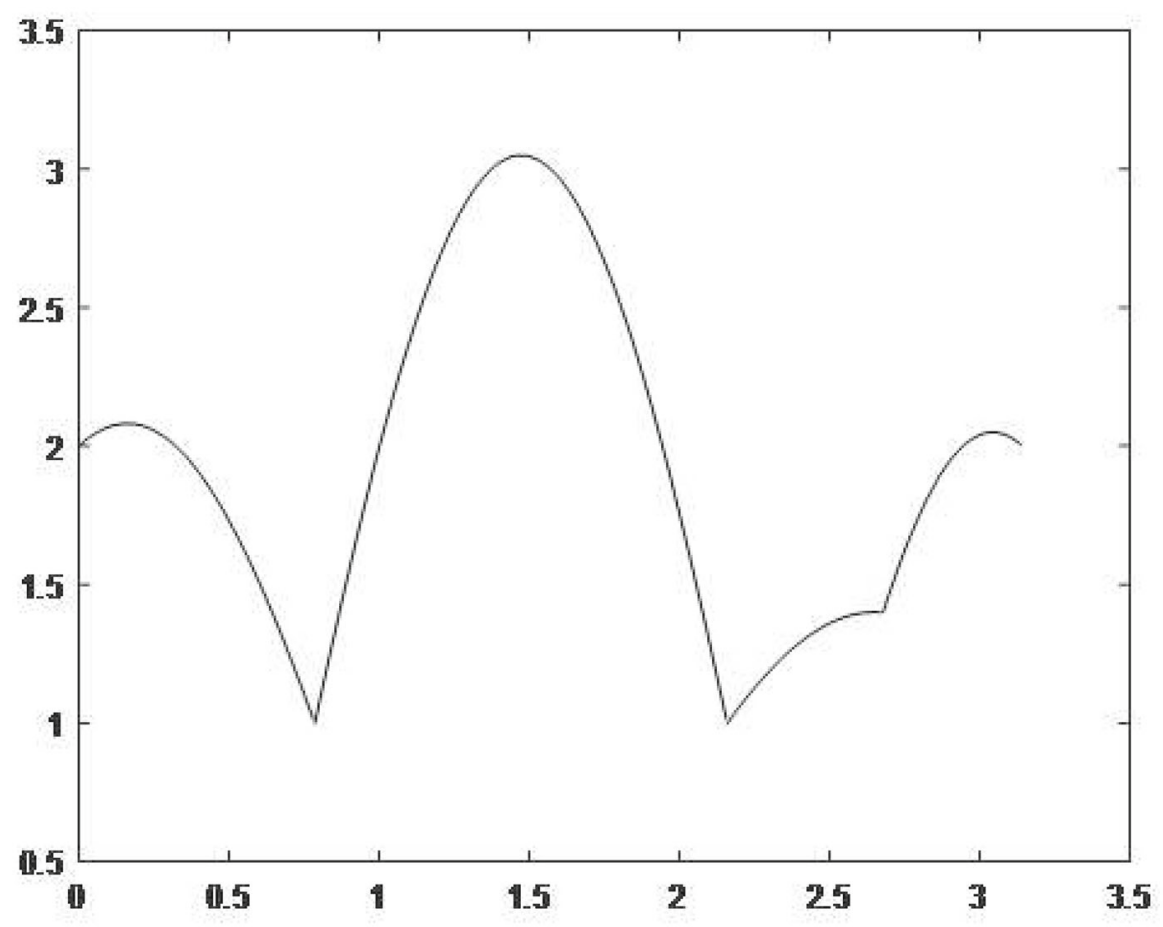

Fig. 1 .

The following lemmas are useful at order to see how tight the lower bound of next Theorem 3 is. See also Example 3.

Lemma $2 \sup _{H \in \mathcal{H}}\left\|P_{H}\right\|_{*}$ is attained at some one-dimensional $\widehat{H} \in \mathcal{H}$.

Proof Let us write $\sup _{H \in \mathcal{H}}\left\|P_{H}\right\|_{*}=\lim _{r \rightarrow \infty}\left\|P_{H_{r}}\right\|_{*}$ for an appropriate sequence of linear subspaces $\left\{H_{r}\right\}_{r \in \mathbb{N}}$. For each $r$ pick $w_{r} \in$ bd $B_{*}$ and $\widehat{w}_{r}:=P_{H_{r}} w_{r}$ with $\left\|\widehat{w}_{r}\right\|_{*}=\left\|P_{H_{r}}\right\|_{*}$.

It is not restrictive to assume (by taking suitable subsequences) that $w_{r} \rightarrow w$ and $\widehat{w}_{r} \rightarrow \widehat{w}$ for some $w \in$ bd $B_{*}$ and some $\widehat{w} \in \mathbb{R}^{n}$ with $\|\widehat{w}\|_{*}=\sup _{H \in \mathcal{H}}\left\|P_{H}\right\|_{*}$. Take $\widehat{H}:=\operatorname{span}\{\widehat{w}\}$ and let us see that $\widehat{w}=P_{\widehat{H}} w$. Indeed,

$$
\widehat{w}^{\prime}(w-\widehat{w})=\lim _{r \rightarrow \infty} \widehat{w}_{r}^{\prime}\left(w_{r}-\widehat{w}_{r}\right)=\lim _{r \rightarrow \infty} 0=0 .
$$

Lemma 3 There exists a one-dimensional $\widetilde{H} \in \mathcal{H}$ such that $\left\|P_{\widetilde{H}}\right\|_{*}=1$.

Proof Take any $\widetilde{w} \in$ bd $B_{*}$ such that $\|\widetilde{w}\|_{2}=\max _{w \in B_{*}}\|w\|_{2}$ and let $\widetilde{H}:=\operatorname{span}\{\widetilde{w}\}$. Then, for any $w \in B_{*}$ there exists $\lambda_{w} \geq 0$ such that $P_{\widetilde{H}} w=\lambda_{w} \widetilde{w}$, and the choice of $\widetilde{w}$ yields

$$
\lambda_{w}\|\widetilde{w}\|_{2}=\left\|P_{\widetilde{H}} w\right\|_{2} \leq\|w\|_{2} \leq\|\widetilde{w}\|_{2} .
$$


Consequently, $\left\|P_{\widetilde{H}} w\right\|_{*}=\lambda w \leq 1$ for all $w \in B_{*}$.

\section{A projection-based approach to the Lipschitz modulus under partial perturbations}

In this section, we consider separately $c$-perturbations, with $\bar{b}$ fixed, and $b$ perturbations, with $\bar{c}$ fixed.

\subsection{Local c-perturbations}

Now we provide in Theorem 3 the announced new lower bound on $\operatorname{lip} \vartheta_{\bar{b}}(\bar{c})$, which may be greater or less than $d\left(0_{n}, \mathcal{F}^{o p}(\bar{\pi})\right.$ ) (see Theorem 1), as we will see in Example 4. Moreover, Example 3 shows that, for any given norm in $\mathbb{R}^{n}$, the upper bound in Theorem 1 and the worst-case of lower bounds in Theorem 3 may be attained for appropriate choices of the given data $\left(\bar{a}_{t}\right)_{t \in T}$. First, we need a technical lemma, which follows from standard arguments in LP. Nevertheless, for the sake of completeness, we give a proof. Recall that $\mathcal{F}(\bar{b})=\left\{x \in \mathbb{R}^{n}: \bar{a}_{t}^{\prime} x \leq \bar{b}_{t}, t \in T=\{1, \ldots, m\}\right\}$.

Lemma 4 Let $\bar{x}$ be an extreme point of the polyhedral set $F:=\mathcal{F}(\bar{b}) \cap$ span $\left\{\bar{a}_{t}, t \in T\right\}$. Then, there exist $\bar{u} \in \mathbb{R}^{n}$ and $\varepsilon>0$ such that $u^{\prime} \bar{x} \leq u^{\prime} x$ for all $x \in F$ and all $u \in \mathbb{R}^{n}$ satisfying $\|u-\bar{u}\|<\varepsilon$ (with $\|\cdot\|$ being any given norm in $\mathbb{R}^{n}$ ).

Proof Let $H=\operatorname{span}\left\{\bar{a}_{t}, t \in T\right\}$ and write $H=\left\{x \in \mathbb{R}^{n}: q_{i}^{\prime} x=0, i \in I\right\}$ for some finite index set $I$ and some $\left(q_{i}\right)_{i \in I} \in\left(\mathbb{R}^{n}\right)^{I}$.

Since $\bar{x} \in \operatorname{extr} \mathcal{F}(\bar{b}) \cap H$, there exists $J \subset T$ such that $a_{t}^{\prime} \bar{x}=\bar{b}_{t}$ for all $t \in J$ and $\operatorname{span}\left(\left\{\bar{a}_{t}, t \in J\right\} \cup\left\{q_{i}, i \in I\right\}\right)=\mathbb{R}^{n}$ (see [2, Theorems 2.2 and 2.3.]). Let

$$
Z:=\text { cone }\left(\left\{-\bar{a}_{t}, t \in J\right\} \cup\left\{q_{i}, i \in I\right\}\right),
$$

thus int $Z \neq \emptyset$. Choose any $\bar{u} \in \operatorname{int} Z$. Then, there is some $\varepsilon>0$ such that $U:=\{u \in$ $\left.\mathbb{R}^{n}:\|u-\bar{u}\|_{*}<\varepsilon\right\}$ is a subset of $Z$. Hence,

$$
u^{\prime} \bar{x} \leq u^{\prime} x \text { for all } x \in F \text { and all } u \in U
$$

since each $u \in Z$ has a representation $u=-\sum_{t \in J} \lambda_{t} \bar{a}_{t}+\sum_{i \in I} \mu_{i} q_{i}$ with $\lambda_{t} \geq 0$ $(t \in J)$ and $\mu_{i} \geq 0(i \in I)$, and so, by taking $u^{\prime} x=-\sum_{t \in J} \lambda_{t} \bar{a}_{t}^{\prime} x$ for $x \in \mathcal{F}(\bar{b}) \cap H$ into account,

$$
u^{\prime} \bar{x}=-\sum_{t \in J} \lambda_{t} \bar{a}_{t}^{\prime} \bar{x}=-\sum_{t \in J} \lambda_{t} \bar{b}_{t} \leq-\sum_{t \in J} \lambda_{t} \bar{a}_{t}^{\prime} x=u^{\prime} x,
$$

yielding the thesis of the lemma.

Theorem 3 Let $\bar{\pi} \in \operatorname{dom} \mathcal{F}^{o p}$. Then,

$$
\operatorname{lip} \vartheta_{\bar{b}}^{R}(\bar{c}) \geq \frac{e\left(\mathcal{E}^{o p}(\bar{\pi}), 0_{n}\right)}{\|P\|_{*}},
$$


where $\|P\|_{*}:=\max _{\|u\|_{*}=1}\|P u\|_{*}$ and $P$ stands for the orthogonal projection on the subspace $\operatorname{span}\left\{\bar{a}_{t}, t \in T\right\}$ with respect to the usual inner product in $\mathbb{R}^{n}$.

Proof Take $\bar{x} \in \mathcal{E}^{o p}(\bar{\pi})$ with $\|\bar{x}\|=e\left(\mathcal{E}^{o p}(\bar{\pi}), 0_{n}\right)$. Then, $\bar{x}$ is also an extreme point of the polyhedral set $\mathcal{F}(\bar{b}) \cap \operatorname{span}\left\{\bar{a}_{t}, t \in T\right\}$ (recall (3) and (4), and the characterization of extreme points in [2, Theorems 2.2 and 2.3.]). Thus, by applying the previous lemma (with taking the dual norm $\|\cdot\|_{*}$ ), there exist $\bar{u} \in \mathbb{R}^{n}$ and $\varepsilon>0$ such that $u^{\prime} \bar{x} \leq u^{\prime} x$ for all $x \in \mathcal{F}(\bar{b}) \cap \operatorname{span}\left\{\bar{a}_{t}, t \in T\right\}$ and all $u \in \mathbb{R}^{n}$ satisfying $\|u-\bar{u}\|_{*}<\varepsilon$.

Let $w \in \mathbb{R}^{n}$ with $\|w\|_{*}=1$ be such that $w^{\prime} \bar{x}=\|\bar{x}\|$ and let $\widehat{w}:=P w$. For each $r \in \mathbb{N}$ let $c^{r}:=\bar{c}+\frac{1}{r} \bar{u}$ and $\widetilde{c}^{r}:=c^{r}+\frac{1}{r^{2}} \widehat{w}$. Then, we have $c^{r}, \widetilde{c}^{r} \rightarrow \bar{c}$ and we claim that

$$
\bar{x} \in \mathcal{F}^{o p}\left(c^{r}, \bar{b}\right) \cap \mathcal{F}^{o p}\left(\widetilde{c}^{r}, \bar{b}\right) \text { whenever }\left\|\frac{1}{r} \widehat{w}\right\|_{*}<\varepsilon
$$

In fact, to see $\bar{x} \in \mathcal{F}^{o p}\left(\widetilde{c}^{r}, \bar{b}\right)$ for such an $r$, we observe that for all $x \in \mathcal{F}(\bar{b})$ and all $v \in \operatorname{span}\left\{\bar{a}_{t}, t \in T\right\}$, one has $v^{\prime} x=v^{\prime} P x$ and, therefore, since $P x \in \mathcal{F}(\bar{b}) \cap$ $\operatorname{span}\left\{\bar{a}_{t}, t \in T\right\}$,

$$
\begin{aligned}
\left(\widetilde{c}^{r}\right)^{\prime} x & =\bar{c}^{\prime} x+\frac{1}{r}\left(\bar{u}+\frac{1}{r} \widehat{w}\right)^{\prime} x=\bar{c}^{\prime} x+\frac{1}{r}\left(\bar{u}+\frac{1}{r} \widehat{w}\right)^{\prime} P x \\
& \geq \bar{c}^{\prime} \bar{x}+\frac{1}{r}\left(\bar{u}+\frac{1}{r} \widehat{w}\right)^{\prime} \bar{x}=\left(\widetilde{c}^{r}\right)^{\prime} \bar{x} .
\end{aligned}
$$

The proof of $\bar{x} \in \mathcal{F}^{o p}\left(c^{r}, \bar{b}\right)$ is similar (by just replacing $\widehat{w}$ with $\left.0_{n}\right)$. Consequently,

$$
\begin{aligned}
\operatorname{lip} \vartheta_{\bar{b}}^{R}(\bar{c}) & \geq \lim _{r \rightarrow \infty} \frac{\vartheta\left(\widetilde{c}^{r}, \bar{b}\right)-\vartheta\left(c^{r}, \bar{b}\right)}{\left\|\widetilde{c}^{r}-c^{r}\right\|_{*}}=\lim _{r \rightarrow \infty} \frac{\left(\widetilde{c}^{r}-c^{r}\right)^{\prime} \bar{x}}{\left\|\widetilde{c}^{r}-c^{r}\right\|_{*}} \\
& =\frac{\widehat{w}^{\prime} \bar{x}}{\|\widehat{w}\|_{*}}=\frac{w^{\prime} \bar{x}}{\|\widehat{w}\|_{*}} \geq \frac{\|\bar{x}\|}{\|P\|_{*}},
\end{aligned}
$$

where we used $\left\|\widehat{c}^{r}-c^{r}\right\|_{*}^{-1}\left(\widehat{c}^{r}-c^{r}\right)=\|\widehat{w}\|_{*}^{-1} \widehat{w}, \widehat{w}^{\prime} \bar{x}=\bar{x}^{\prime} P w=\bar{x}^{\prime} w=w^{\prime} \bar{x}=\|\bar{x}\|$ and $\|P\|_{*} \geq\|P w\|_{*}=\|\widehat{w}\|_{*}$.

Corollary 1 If $\|P\|_{*}=1$, for instance when $\|\cdot\|$ is the Euclidean norm or $\operatorname{span}\left\{\bar{a}_{t}, t \in\right.$ $T\}=\mathbb{R}^{n}$, we have $\operatorname{lip} \vartheta_{\bar{b}}^{R}(\bar{c})=e\left(\mathcal{E}^{o p}(\bar{\pi}), 0_{n}\right)$. In the particular case when $\bar{c} \in \operatorname{int} C$, we can write $\operatorname{lip} \vartheta_{\bar{b}}^{R}(\bar{c})=e\left(\mathcal{F}^{o p}(\bar{\pi}), 0_{n}\right)$.

Example 3 For any given norm $\|\cdot\|$ in $\mathbb{R}^{n}$, let $\widehat{w}$ and $\widetilde{w}$ be as in the proof of Lemmas 2 and 3 , respectively; and consider the next two problems $\widehat{\pi}=(\widehat{c}, \widehat{b}), \widetilde{\pi}=(\widetilde{c}, \widetilde{b}) \in$ $\operatorname{dom} \mathcal{F}^{o p} \subset \mathbb{R}^{n} \times \mathbb{R}$, with one single constraint each (i.e., $T=\{1\}$ ):

$$
\begin{aligned}
& \widehat{\pi}: \text { Inf } \widehat{w}^{\prime} x \text { subject to }-\widehat{w}^{\prime} x \leq-\|\widehat{w}\|_{2}^{2}, \\
& \tilde{\pi}: \operatorname{Inf} \widetilde{w}^{\prime} x \text { subject to }-\widetilde{w}^{\prime} x \leq-\|\widetilde{w}\|_{2}^{2} .
\end{aligned}
$$


Then, the associated moduli (note that the left-hand sides are different) are

$$
\operatorname{lip} \widehat{\vartheta}_{\widehat{b}}^{R}(\widehat{c})=\frac{e\left(\mathcal{E}^{o p}(\widehat{\pi}), 0_{n}\right)}{\left\|P_{\widehat{H}}\right\|_{*}} \text { and } \operatorname{lip} \widetilde{\vartheta}_{\widetilde{b}}^{R}(\widetilde{c})=e\left(\mathcal{E}^{o p}(\widetilde{\pi}), 0_{n}\right)
$$

where, as in the proof of Lemma 2,

$$
\widehat{H}=\operatorname{span}\{\widehat{w}\} \text { and }\left\|P_{\widehat{H}}\right\|_{*}=\max _{H \in \mathcal{H}}\left\|P_{H}\right\|_{*}
$$

Indeed, it is clear that $\mathcal{F}^{o p}(\widehat{\pi})=\widehat{w}+\{\widehat{w}\}^{\perp}$, so that $\mathcal{E}^{o p}(\widehat{\pi})=\{\widehat{w}\}$. Accordingly, since $\left(\|\cdot\|_{*}\right)_{*}=\|\cdot\|, P_{\widehat{H}} u=\left[\left(\widehat{w} /\|\widehat{w}\|_{2}\right)^{\prime} u\right]\left(\widehat{w} /\|\widehat{w}\|_{2}\right)$ for all $u \in \mathbb{R}^{n}$, and recalling from the proof of Lemma 2 that $\widehat{w}=P_{\widehat{H}} w$ for some $w \in B_{*}$, one has

$$
\begin{aligned}
e\left(\mathcal{E}^{o p}(\widehat{\pi}), 0_{n}\right) & =\|\widehat{w}\|=\max _{u \in B_{*}} \widehat{w}^{\prime} u=\max _{u \in B_{*}} \widehat{w}^{\prime} P_{\widehat{H}} u \\
& \geq \widehat{w}^{\prime} P_{\widehat{H}} w=\widehat{w}^{\prime} \widehat{w}=\|\widehat{w}\|_{2}^{2} .
\end{aligned}
$$

We can directly compute $\operatorname{lip} \widehat{\vartheta} \widehat{b}_{\widehat{b}}^{R}(\widehat{c})$ by observing that $\widehat{c}=\widehat{w}$ and $\widehat{b}=-\|\widehat{w}\|_{2}^{2}$, and a perturbed $\pi=(c, \widehat{b})$ belongs to $\operatorname{dom} \mathcal{F}^{o p}$ if and only if $c=\alpha \widehat{w}$ for some $\alpha \geq 0$. Therefore,

$$
\begin{aligned}
\operatorname{lip} \widehat{\vartheta}_{\widehat{b}}^{R}(\widehat{c}) & =\limsup _{\alpha_{1}, \alpha_{2} \rightarrow 1, \alpha_{1} \neq \alpha_{2}} \frac{\left|\widehat{\vartheta}_{\widehat{b}}^{R}\left(\alpha_{1} \widehat{w}\right)-\widehat{\vartheta} \widehat{\vartheta}_{\widehat{b}}^{R}\left(\alpha_{2} \widehat{w}\right)\right|}{\left\|\alpha_{1} \widehat{w}-\alpha_{2} \widehat{w}\right\|_{*}} \\
& =\limsup _{\alpha_{1}, \alpha_{2} \rightarrow 1, \alpha_{1} \neq \alpha_{2}} \frac{\left|\alpha_{1} \widehat{w}^{\prime} \widehat{w}-\alpha_{2} \widehat{w}^{\prime} \widehat{w}\right|}{\left|\alpha_{1}-\alpha_{2}\right|\|\widehat{w}\|_{*}}=\frac{\|\widehat{w}\|_{2}^{2}}{\|\widehat{w}\|_{*}} \leq \frac{e\left(\mathcal{E}^{o p}(\widehat{\pi}), 0_{n}\right)}{\left\|P_{\widehat{H}}\right\|_{*}} .
\end{aligned}
$$

Finally, Theorem 3 provides the converse inequality.

On the other hand, the case of $\operatorname{lip} \widetilde{\vartheta}_{\widetilde{b}}^{R}(\widetilde{c})$ is sensibly easier since $\left\|P_{\widetilde{H}}\right\|_{*}=1$ and then Theorems 1 and 3 give the aimed equality.

Putting together Theorems 1 and 3, we have the next corollary.

Corollary 2 Let $\bar{\pi} \in \operatorname{dom} \mathcal{F}^{o p}$. Then,

$$
\max \left\{d\left(0_{n}, \mathcal{F}^{o p}(\bar{\pi})\right), \frac{e\left(\mathcal{E}^{o p}(\bar{\pi}), 0_{n}\right)}{\|P\|_{*}}\right\} \leq \operatorname{lip} \vartheta_{\bar{b}}(\bar{c}) \leq e\left(\mathcal{E}^{o p}(\bar{\pi}), 0_{n}\right)
$$

The next example shows that any of both lower bounds on $\operatorname{lip} \vartheta \frac{R}{b}(\bar{c})$, namely $d\left(0_{n}, \mathcal{F}^{o p}(\bar{\pi})\right)$ and $\frac{e\left(\mathcal{E}^{o p}(\bar{\pi}), 0_{n}\right)}{\|P\|_{*}}$, may be greater or less than the other.

Example 4 Consider $\mathbb{R}^{3}$ endowed with the norm given by

$$
\|x\|:=\left|x_{2}\right|+\max \left\{\left|2 x_{1}+x_{3}\right|,\left|2 x_{1}+3 x_{3}\right|\right\},
$$


whose dual norm $\|\cdot\|_{*}$ has as its closed unit ball the set

$$
B_{*}:=\operatorname{conv}\left\{(-2, \pm 1,-1)^{\prime},(-2, \pm 1,-3)^{\prime},(2, \pm 1,1)^{\prime},(2, \pm 1,3)^{\prime}\right\}
$$

Alternatively, we may start by considering $B_{*}$ and obtain $\|\cdot\|$ as $\left(\|\cdot\|_{*}\right)_{*}$. Consider the problems in $\mathbb{R}^{3}$

$$
\begin{aligned}
\bar{\pi}_{1}: \text { minimize } & x_{1} \\
\text { subject to } & -x_{1} \leq 0 \\
& -x_{2} \leq-1 .
\end{aligned}
$$

$$
\begin{aligned}
\bar{\pi}_{2}: \text { minimize } & x_{1} \\
\text { subject to } & -x_{1} \leq 0 \\
& -x_{2} \leq-1 \\
& x_{2} \leq 3 .
\end{aligned}
$$

Note that $\|P\|_{*}=2$ and it can be easily checked that $\mathcal{E}^{o p}\left(\bar{\pi}_{1}\right)=\left\{(0,1,0)^{\prime}\right\}$ and $\mathcal{E}^{o p}\left(\bar{\pi}_{2}\right)=\left\{(0,1,0)^{\prime},(0,3,0)^{\prime}\right\}$. Then, we have

$$
\frac{e\left(\mathcal{E}^{o p}\left(\bar{\pi}_{1}\right), 0_{3}\right)}{\|P\|_{*}}=\frac{1}{2}<1=d\left(0_{3}, \mathcal{F}^{o p}\left(\bar{\pi}_{1}\right)\right),
$$

and

$$
\frac{e\left(\mathcal{E}^{o p}\left(\bar{\pi}_{2}\right), 0_{3}\right)}{\|P\|_{*}}=\frac{3}{2}>1=d\left(0_{3}, \mathcal{F}^{o p}\left(\bar{\pi}_{2}\right)\right) .
$$

\subsection{Local $b$-perturbations}

This short subsection basically consists of a reformulation of known results. Given $\bar{\pi}=(\bar{c}, \bar{b}) \in \operatorname{dom} \mathcal{F}^{o p}$, it is well known that for each $(\bar{c}, b) \in \operatorname{dom} \mathcal{F}^{o p}$ the optimal value $\vartheta_{\bar{c}}^{R}(b)$ coincides with that of the dual problem

$$
\pi^{D}: \text { Sup }-b^{\prime} \lambda \text { subject to } \sum_{t=1}^{m} \lambda_{t} \bar{a}_{t}=-\bar{c}, \lambda \geq 0_{m},
$$

where $\lambda=\left(\lambda_{t}\right)_{t=1}^{m} \in \mathbb{R}^{m}$ is the decision variable. Observe that this subsection considers a fixed $\bar{c} \in C$ (recall (2)), which entails the feasibility of $\pi^{D}$ for any $b \in \mathbb{R}^{m}$. Hence, by duality theory, the boundedness (equivalently, solvability) of $\pi^{D}$ is equivalent to primal feasibility. Formally, denoting by $\Lambda^{o p}(\bar{c}, b)$ the optimal set of the problem $\pi^{D}$, one has $\operatorname{dom} \Lambda^{o p}(\bar{c}, \cdot)=\operatorname{dom} \mathcal{F}$. Observe that, viewed as a parameter, $\bar{\pi}^{D}$ is, as well as $\bar{\pi}$, identified with $(\bar{c}, \bar{b})$.

Recalling Remark 3, [5, Theorem 3.1] provides an exact point-based expression for $\operatorname{lip} \vartheta_{\bar{c}}^{R}(\bar{b})$. This expression is given in terms of the extreme points of the optimal set of $\bar{\pi}^{D}$ (see [4, Lemma 1]). More specifically, denoting by $\|\cdot\|_{1}$ the $l_{1}$-norm in $\mathbb{R}^{n}$, dual to $\|\cdot\|_{\infty}$ (used for $b$-perturbations), [5, Theorem 3.1] can be reformulated as

$$
\operatorname{lip} \vartheta_{\bar{c}}^{R}(\bar{b})=e_{1}\left(\operatorname{extr} \Lambda^{o p}(\bar{\pi}), 0_{m}\right),
$$


where we have written $e_{1}$ to emphasize that the Hausdorff excess is considered with respect to $\|\cdot\|_{1}$.

Remark 4 The constraint $\lambda \geq 0_{m}$ in the formulation of $\bar{\pi}^{D}$, translated into the primal terminology ensures that $\bar{\pi}^{\bar{D}}$ satisfies the counterpart of condition $\operatorname{span}\left\{\bar{a}_{t}, t \in T\right\}=$ $\mathbb{R}^{n}$. Accordingly, (9) is directly derived from Corollary 1.

\subsection{Global Lipschitz moduli}

In the next paragraphs, we appeal to the following sets associated with $b \in \operatorname{dom} \mathcal{F}$ and $c \in C$, respectively:

$$
\begin{aligned}
& \mathcal{E}(b):=\operatorname{extr}\left(\mathcal{F}(b) \cap \operatorname{span}\left\{\bar{a}_{t}, t \in T\right\}\right), \\
& \Lambda(c):=\left\{\lambda \in \mathbb{R}_{+}^{m}:-c=\sum_{t=1}^{m} \lambda_{t} \bar{a}_{t}\right\} .
\end{aligned}
$$

The last one is indeed the feasible set of the dual problem of $\pi$. A global result can be obtained from local ones by focusing on the origin.

Theorem 4 Let $\bar{\pi}=(\bar{c}, \bar{b}) \in \operatorname{dom} \mathcal{F}^{o p}$. Then,

$$
\begin{aligned}
& \text { (i) } \frac{e\left(\mathcal{E}(\bar{b}), 0_{n}\right)}{\|P\|_{*}} \leq \operatorname{g}-\operatorname{lip} \vartheta_{\bar{b}}^{R} \leq e\left(\mathcal{E}(\bar{b}), 0_{n}\right)=\max _{x \in \mathcal{E}(\bar{b})}\|x\| ; \\
& \text { (ii) } \operatorname{g-lip} \vartheta_{\bar{c}}^{R}=e_{1}\left(\operatorname{extr} \Lambda(\bar{c}), 0_{m}\right)=\max _{\lambda \in \Lambda(\bar{c})}\|\lambda\|_{1} .
\end{aligned}
$$

Proof Just observe that $\mathcal{F}(\bar{b})=\mathcal{F}^{o p}\left(0_{n}, \bar{b}\right)$ and $\mathcal{E}(\bar{b})=\mathcal{E}^{o p}\left(0_{n}, \bar{b}\right)$, so that the result will follow from Corollary 2 if we prove that $\mathrm{g}$-lip $\vartheta_{\bar{b}} \leq \operatorname{lip} \vartheta_{\bar{b}}\left(0_{n}\right)$ (the converse inequality is obvious from the definitions). Pick arbitrarily $\varepsilon>0$ and, by the definition of Lipschitz modulus, take $\delta>0$ such that

$$
\left.\underset{c, \tilde{c} \in C}{\|c\|_{*},\|\widetilde{c}\|_{*}<\delta}\right\} \Rightarrow|\vartheta(c, \bar{b})-\vartheta(\widetilde{c}, \bar{b})| \leq\left(\operatorname{lip} \vartheta_{\bar{b}}^{R}\left(0_{n}\right)+\varepsilon\right)\|c-\widetilde{c}\|_{*} .
$$

Now consider any $c, \tilde{c} \in C$ and take $\mu>0$ such that $\|\mu c\|_{*},\|\mu \widetilde{c}\|_{*}<\delta$. Then, (10) yields

$$
\begin{aligned}
|\vartheta(c, \bar{b})-\vartheta(\widetilde{c}, \bar{b})| & =\left|\mu^{-1} \vartheta(\mu c, \bar{b})-\mu^{-1} \vartheta(\mu \widetilde{c}, \bar{b})\right| \\
& \leq \mu^{-1}\left(\operatorname{lip} \vartheta_{\bar{b}}^{R}\left(0_{n}\right)+\varepsilon\right)\|\mu c-\mu \widetilde{c}\|_{*} \\
& =\left(\operatorname{lip} \vartheta_{\bar{b}}^{R}\left(0_{n}\right)+\varepsilon\right)\|c-\widetilde{c}\|_{*}
\end{aligned}
$$

Since this happens for all $\varepsilon>0$, ( $i$ ) follows.

The proof of $(i i)$ is completely analogous, working with the dual problem $\pi^{D}$ (see (8)), replacing $c \in C$ with $b \in \operatorname{dom} \mathcal{F}$, using $\|b\|_{\infty}$ instead of $\|c\|_{*}$ and taking the beginning of Remark 4 into account. 


\section{Lipschitz modulus under canonical perturbations}

In this section, we combine some ideas of the previous ones to study local and global Lipschitz moduli under canonical perturbations.

\subsection{Local perturbations}

The next theorem can be seen as the counterpart of Theorem 3 for canonical perturbations. It appeals to the following lemma which can be partially traced out from [4, Lemma 2] (see also references therein) written in terms of limits of sequences of subsets in the Painlevé-Kuratowski sense. In order to distinguish this type of limits (of sets) from ordinary limits of points, we use the symbol ' $\operatorname{Lim}_{r \rightarrow \infty}$ ' instead of ' $\lim _{r \rightarrow \infty}$ '. In the sequel, $T_{b}(x)$ represents the set of active indices at $x \in \mathcal{F}(b)$, for $b \in \mathbb{R}^{T}$, i.e.,

$$
T_{b}(x):=\left\{t \in T \mid \bar{a}_{t}^{\prime} x=b_{t}\right\}
$$

Lemma 5 Let $\bar{\pi}=(\bar{c}, \bar{b}) \in \operatorname{dom} \mathcal{F}^{o p}$ and $\left\{b^{r}\right\}_{r \in \mathbb{N}} \subset \operatorname{dom} \mathcal{F}$ converging to $\bar{b}$. Then,

(i) $\left\{\mathcal{E}\left(b^{r}\right)\right\}_{r \in \mathbb{N}}$ is uniformly bounded and $\emptyset \neq \operatorname{Lim}_{r \rightarrow \infty} \mathcal{E}\left(b^{r}\right)=\mathcal{E}(\bar{b})$;

(ii) $\left\{\mathcal{E}^{o p}\left(\bar{c}, b^{r}\right)\right\}_{r \in \mathbb{N}}$ is uniformly bounded and

$$
\emptyset \neq \operatorname{Lim}_{r \rightarrow \infty} \mathcal{E}^{o p}\left(\bar{c}, b^{r}\right)=\mathcal{E}^{o p}(\bar{\pi}) .
$$

Proof $(i)$ is exactly as [4, Lemma $2(i)$ ], and (ii) comes from $(i)$ by just taking into account that, for each $r, \mathcal{F}^{o p}\left(\bar{c}, b^{r}\right)$ is nothing else but the feasible set of system $\left\{\bar{c}^{\prime} x \leq \vartheta\left(\bar{c}, b^{r}\right), \bar{a}_{t}^{\prime} x \leq b_{t}^{r}, t \in T\right\}$; recall also the well-known fact that (see, e.g., [4, Theorem 2]) $\lim _{r \rightarrow \infty} \vartheta\left(\bar{c}, b^{r}\right)=\lim _{r \rightarrow \infty} \vartheta(\bar{c}, \bar{b})$.

Theorem 5 Let $\bar{\pi}=(\bar{c}, \bar{b}) \in \operatorname{dom} \mathcal{F}^{o p}$. Then,

$$
\operatorname{lip} \vartheta^{R}(\bar{\pi}) \geq \operatorname{lip} \vartheta_{\bar{c}}^{R}(\bar{b})+\frac{e\left(\mathcal{E}^{o p}(\bar{\pi}), 0_{n}\right)}{\|P\|_{*}} .
$$

Proof Let us consider two sequences $\left\{b^{r}\right\}_{r \in \mathbb{N}},\left\{\tilde{b}^{r}\right\}_{r \in \mathbb{N}} \subset$ dom $\mathcal{F}$ converging to $\bar{b}$ such that

$$
\operatorname{lip} \vartheta_{\bar{c}}^{R}(\bar{b})=\lim _{r \rightarrow \infty} \frac{\vartheta_{\bar{c}}\left(\tilde{b}^{r}\right)-\vartheta_{\bar{c}}\left(b^{r}\right)}{\left\|\widetilde{b}^{r}-b^{r}\right\|_{\infty}} .
$$

We can confine ourselves to the case $\operatorname{lip} \vartheta_{\bar{c}}(\bar{b})>0$, since otherwise the thesis of the present theorem follows directly from Theorem 3. Accordingly, we may assume $\vartheta_{\bar{c}}\left(\widetilde{b}^{r}\right)-\vartheta_{\bar{c}}\left(b^{r}\right)>0$ for $r$ large enough. In particular, this implies $\bar{c} \neq 0$. This detail will be used later.

Take $\bar{x} \in \mathcal{E}^{o p}(\bar{\pi})$ with $\|\bar{x}\|=e\left(\mathcal{E}^{o p}(\bar{\pi}), 0_{n}\right)$. We may assume $\bar{x} \neq 0_{n}$ (otherwise the thesis of the theorem is trivial). The previous lemma entails $\mathcal{E}^{o p}(\bar{\pi})=$ 
$\operatorname{Lim}_{r \rightarrow \infty} \mathcal{E}^{o p}\left(\bar{c}, \widetilde{b}^{r}\right)$. In particular, there exists a sequence $\left\{\widetilde{x}^{r}\right\}_{r \in \mathbb{N}}$ converging to $\bar{x}$ with $\tilde{x}^{r} \in \mathcal{E}^{o p}\left(\bar{c}, \tilde{b}^{r}\right)$ for all $r \in \mathbb{N}$. According to the KKT conditions, we have $-\bar{c} \in$ cone $\left\{\bar{a}_{t}, t \in T_{\widetilde{b}^{r}}\left(\widetilde{x}^{r}\right)\right\}$ for all $r \in \mathbb{N}$. By virtue of Carathéodory's Theorem, for each $r$ there exists a subset $D^{r} \subset T_{\widetilde{b}^{r}}\left(\widetilde{x}^{r}\right)$ with $-\bar{c} \in$ cone $\left\{\bar{a}_{t}, t \in D^{r}\right\}$ and such that $\left\{\bar{a}_{t}, t \in D^{r}\right\}$ is linearly independent. For each $r$, since $\tilde{x}^{r} \in \mathcal{E}^{o p}\left(\bar{c}, \widetilde{b}^{r}\right)$, we have $\operatorname{span}\left\{\bar{a}_{t}, t \in T_{\widetilde{b}^{r}}\left(\widetilde{x}^{r}\right)\right\}=\operatorname{span}\left\{\bar{a}_{t}, t \in T\right\}$, which entails that $\left\{\bar{a}_{t}, t \in D^{r}\right\}$ can be enlarged to a basis $\left\{\bar{a}_{t_{1}^{r}}, \ldots, \bar{a}_{t_{k}^{r}}\right\}$ of span $\left\{\bar{a}_{t}, t \in T\right\}$ with $\left\{t_{1}^{r}, \ldots, t_{k}^{r}\right\} \subset T_{\widetilde{b}^{r}}\left(\widetilde{x}^{r}\right) \subset T$. Since $T$ is finite, we may assume without loss of generality (by taking a suitable subsequence) that $\left\{t_{1}^{r}, \ldots, t_{k}^{r}\right\}$ does not depend on $r$, say $\left\{t_{1}^{r}, \ldots, t_{k}^{r}\right\}=\left\{t_{1}, \ldots, t_{k}\right\}$ for all $r$. Clearly $\left\{t_{1}, \ldots, t_{k}\right\} \subset T_{\widetilde{b}^{r}}\left(\widetilde{x}^{r}\right)$ implies $\left\{t_{1}, \ldots, t_{k}\right\} \subset T_{\bar{b}}(\bar{x})$.

Now define

$$
0_{n} \neq \bar{u}:=-\sum_{i=1}^{k} \bar{a}_{t_{i}}
$$

which implies $-\bar{u} \in$ int cone $\left\{\bar{a}_{t_{1}}, \ldots, \bar{a}_{t_{k}}\right\}$ (see [6, Theorem A.7]). Take $\varepsilon>0$ such that

$$
\left[u \in \operatorname{span}\left\{\bar{a}_{t}, t \in T\right\} \text { and }\|u-\bar{u}\|_{*}<\varepsilon\right] \Rightarrow-u \in \text { cone }\left\{\bar{a}_{t_{1}}, \ldots, \bar{a}_{t_{k}}\right\}
$$

In the case when $k:=\operatorname{dim} \operatorname{span}\left\{\bar{a}_{t}, t \in T\right\}<n$, let $\left\{v_{k+1}, \ldots, v_{n}\right\}$ be a basis of $\left\{\bar{a}_{t}, t \in T\right\}^{\perp}$, so that $\left\{\bar{a}_{t_{1}}, \ldots, \bar{a}_{t_{k}}, v_{k+1}, \ldots, v_{n}\right\}$ is a basis of $\mathbb{R}^{n}$. Let $A$ denote the matrix whose rows are $\bar{a}_{t_{1}}^{\prime}, \ldots, \bar{a}_{t_{k}}^{\prime}$ and $Q$ the one whose rows are $v_{k+1}^{\prime}, \ldots, v_{n}^{\prime}$. We have and define

$$
\bar{x}=\left(\begin{array}{c}
A \\
Q
\end{array}\right)^{-1}\left(\begin{array}{c}
\left(\bar{b}_{t_{i}}\right)_{i=1}^{k} \\
0_{n-k}
\end{array}\right), \tilde{x}^{r}=\left(\begin{array}{c}
A \\
Q
\end{array}\right)^{-1}\left(\begin{array}{c}
\left(\widetilde{b}_{t_{i}}^{r}\right)_{i=1}^{k} \\
0_{n-k}
\end{array}\right), x^{r}:=\left(\begin{array}{c}
A \\
Q
\end{array}\right)^{-1}\left(\begin{array}{c}
\left(b_{t_{i}}^{r}\right)_{i=1}^{k} \\
0_{n-k}
\end{array}\right) .
$$

It is clear that $x^{r} \rightarrow \bar{x}$, but some $x^{r}$ may not be feasible for the whole $b^{r}$. Let us define, for each $r \in \mathbb{N}, \beta^{r}, \widetilde{\beta}^{r} \in \mathbb{R}^{T}$ given by

$$
\begin{aligned}
& \beta_{t}^{r}:= \begin{cases}b_{t}^{r} & \text { if } t \in\left\{t_{1}, \ldots, t_{k}\right\}, \\
\max \left\{b_{t}^{r}, \widetilde{b}_{t}^{r}, \bar{a}_{t}^{\prime} x^{r}\right\} & \text { if } t \in T \backslash\left\{t_{1}, \ldots, t_{k}\right\} ;\end{cases} \\
& \widetilde{\beta}_{t}^{r}:= \begin{cases}\widetilde{b}_{t}^{r} & \text { if } t \in\left\{t_{1}, \ldots, t_{k}\right\}, \\
\max \left\{b_{t}^{r}, \widetilde{b}_{t}^{r}, \bar{a}_{t}^{\prime} x^{r}\right\} & \text { if } t \in T \backslash\left\{t_{1}, \ldots, t_{k}\right\} .\end{cases}
\end{aligned}
$$

Obviously, $\left\|\beta^{r}-\widetilde{\beta}^{r}\right\|_{\infty} \leq\left\|b^{r}-\widetilde{b}^{r}\right\|_{\infty}$ and one easily checks

$$
\begin{aligned}
& \max \left\{\left\|\beta^{r}-\bar{b}\right\|_{\infty},\left\|\widetilde{\beta}^{r}-\bar{b}\right\|_{\infty}\right\} \\
\leq & \max \left\{\left\|b^{r}-\bar{b}\right\|_{\infty},\left\|\widetilde{b}^{r}-\bar{b}\right\|_{\infty}, \max _{t \in T}\left|\bar{a}_{t}^{\prime}\left(x^{r}-\bar{x}\right)\right|\right\} \rightarrow 0 .
\end{aligned}
$$

Indeed, the nontrivial case happens for those $t \in T \backslash\left\{t_{1}, \ldots, t_{k}\right\}$ such that $\max \left\{b_{t}^{r}, \widetilde{b}_{t}^{r}\right.$, $\left.\bar{a}_{t}^{\prime} x^{r}\right\}=\bar{a}_{t}^{\prime} x^{r}$. In this case, we distinguish two possibilities: $0 \leq \bar{a}_{t}^{\prime} x^{r}-\bar{b}_{t} \leq$ 
$\bar{a}_{t}^{\prime} x^{r}-\bar{a}_{t}^{\prime} \bar{x}$ or $b_{t}^{r} \leq \bar{a}_{t}^{\prime} x^{r} \leq \bar{b}_{t}$. The first one yields $\left|\bar{a}_{t}^{\prime} x^{r}-\bar{b}_{t}\right| \leq\left|\bar{a}_{t}^{\prime}\left(x^{r}-\bar{x}\right)\right|$ whereas the second one entails $\left|\bar{a}_{t}^{\prime} x^{r}-\bar{b}_{t}\right| \leq\left|b_{t}^{r}-\bar{b}_{t}\right|$.

From the definition, taking into account that, for each $r,\left\{t_{1}, \ldots, t_{k}\right\} \subset T_{\widetilde{b}^{r}}\left(\widetilde{x}^{r}\right) \cap$ $T_{b^{r}}\left(x^{r}\right)$, we straightforwardly obtain $x^{r} \in \mathcal{F}^{o p}\left(\bar{c}, \beta^{r}\right) \cap \mathcal{F}^{o p}\left(\bar{u}, \beta^{r}\right)$ and $\widetilde{x}^{r} \in$ $\mathcal{F}^{o p}\left(\bar{c}, \widetilde{\beta}^{r}\right) \cap \mathcal{F}^{o p}\left(\bar{u}, \widetilde{\beta}^{r}\right)$ (recall (12) and the fact that $-\bar{c} \in$ cone $\left.\left\{\bar{a}_{t_{1}}, \ldots, \bar{a}_{t_{k}}\right\}\right)$. Now, as in the proof of Theorem 3, let $w \in \mathbb{R}^{n}$ with $\|w\|_{*}=1$ be such that $w^{\prime} \bar{x}=\|\bar{x}\|$ and let $\widehat{w}:=P w$. For each $r \in \mathbb{N}$, let us define

$$
\begin{aligned}
c^{r} & :=\bar{c}+\frac{2}{\varepsilon}\left\|\widetilde{\beta}^{r}-\beta^{r}\right\|_{\infty} \bar{u}, \\
\widetilde{c}^{r} & :=\bar{c}+\frac{2}{\varepsilon}\left\|\widetilde{\beta}^{r}-\beta^{r}\right\|_{\infty}\left(\bar{u}+\frac{\varepsilon}{2} \frac{\widehat{w}}{\|\widehat{w}\|_{*}}\right) .
\end{aligned}
$$

We define $\pi^{r}:=\left(c^{r}, \beta^{r}\right)$ and $\tilde{\pi}^{r}:=\left(\widetilde{c}^{r}, \widetilde{\beta}^{r}\right)$, for all $r$. Hence, from (13), $x^{r} \in$ $\mathcal{F}^{o p}\left(\pi^{r}\right)$ and $\tilde{x}^{r} \in \mathcal{F}^{o p}\left(\widetilde{\pi}^{r}\right)$ for each $r$.

Now let us show that $\widetilde{\beta}^{r} \neq \beta^{r}$ holds true, provided that $r$ is large enough to ensure $\vartheta_{\bar{c}}\left(\widetilde{b}^{r}\right)-\vartheta_{\bar{c}}\left(b^{r}\right)>0$ (recall the beginning of the proof). To do this, observe that

$$
(\bar{c})^{\prime} x^{r}=\vartheta\left(\bar{c}, \beta^{r}\right) \leq \vartheta\left(\bar{c}, b^{r}\right) \text { because } \mathcal{F}\left(\beta^{r}\right) \supset \mathcal{F}\left(b^{r}\right) .
$$

Then, since $\widetilde{x}^{r} \in \mathcal{F}^{o p}\left(\bar{c}, \widetilde{b}^{r}\right)$, writing $\bar{c}=-\sum_{i=1}^{k} \lambda_{i} \bar{a}_{t_{i}}$ with $\lambda_{i} \geq 0$ for $i=1, \ldots, k$, we have

$$
\begin{aligned}
0 & <\vartheta_{\bar{c}}\left(\widetilde{b}^{r}\right)-\vartheta_{\bar{c}}\left(b^{r}\right) \leq \bar{c}^{\prime}\left(\widetilde{x}^{r}-x^{r}\right)=-\sum_{i=1}^{k} \lambda_{i} \bar{a}_{t_{i}}^{\prime}\left(\widetilde{x}^{r}-x^{r}\right) \\
& =-\sum_{i=1}^{k} \lambda_{i}\left(\widetilde{\beta}_{t_{i}}-\beta_{t_{i}}\right),
\end{aligned}
$$

which entails $\widetilde{\beta}^{r} \neq \beta^{r}$.

Finally observe that $\left\|\pi^{r}-\widetilde{\pi}^{r}\right\|=\left\|\widetilde{\beta}^{r}-\widetilde{\beta}^{r}\right\|_{\infty}$ because $\left\|\widetilde{c}^{r}-c^{r}\right\|_{*}=\left\|\beta^{r}-\widetilde{\beta}^{r}\right\|_{\infty}$. Thus, recalling $\left\|\beta^{r}-\widetilde{\beta}^{r}\right\|_{\infty} \leq\left\|b^{r}-\widetilde{b}^{r}\right\|_{\infty}$ and (11), we have

$$
\begin{aligned}
& \operatorname{lip} \vartheta^{R}(\bar{\pi}) \geq \limsup _{r \rightarrow \infty} \frac{\vartheta\left(\tilde{\pi}^{r}\right)-\vartheta\left(\pi^{r}\right)}{\left\|\tilde{\pi}^{r}-\pi^{r}\right\|} \\
& =\limsup _{r \rightarrow \infty} \frac{\left(\widetilde{c}^{r}\right)^{\prime} \widetilde{x}^{r}-\left(\widetilde{c}^{r}\right)^{\prime} x^{r}+\left(\widetilde{c}^{r}\right)^{\prime} x^{r}-\left(c^{r}\right)^{\prime} x^{r}}{\left\|\widetilde{\beta}^{r}-\beta^{r}\right\|_{\infty}} \\
& \geq \limsup _{r \rightarrow \infty}\left(\frac{(\bar{c})^{\prime} \widetilde{x}^{r}-(\bar{c})^{\prime} x^{r}-\left\|\widetilde{c}^{r}-\bar{c}\right\|_{*}\left\|\widetilde{x}^{r}-x^{r}\right\|}{\left\|\widetilde{\beta}^{r}-\beta^{r}\right\|_{\infty}}+\frac{\left(\widetilde{c}^{r}-c^{r}\right)^{\prime} x^{r}}{\left\|\widetilde{\beta}^{r}-\beta^{r}\right\|_{\infty}}\right) \\
& \geq \limsup _{r \rightarrow \infty}\left(\frac{\vartheta_{\bar{c}}\left(\widetilde{b}^{r}\right)-\vartheta_{\bar{c}}\left(b^{r}\right)}{\left\|\widetilde{b}^{r}-b^{r}\right\|_{\infty}}-\left(\frac{2}{\varepsilon}\|\bar{u}\|_{*}+1\right)\left\|\widetilde{x}^{r}-x^{r}\right\|+\frac{\widehat{w}^{\prime} x^{r}}{\|\widehat{w}\|_{*}}\right) \\
& =\operatorname{lip} \vartheta_{\bar{c}}^{R}(\bar{b})+\frac{\widehat{w}^{\prime} \bar{x}}{\|\widehat{w}\|_{*}} \geq \operatorname{lip} \vartheta_{\bar{c}}^{R}(\bar{b})+\frac{\|\bar{x}\|}{\|P\|_{*}},
\end{aligned}
$$


where we have taken into account that $\widehat{w}^{\prime} \bar{x}=\|\bar{x}\|$ and that $\|\widehat{w}\|_{*} \leq\|P\|_{*}$.

Putting together the previous theorem with [5, Theorems 3.1 and 5.1] and Theorem 2, we obtain the following result.

Corollary 3 Let $\bar{\pi} \in \operatorname{dom} \mathcal{F}^{o p}$. Then,

$$
\begin{aligned}
& \quad \operatorname{lip} \vartheta_{\bar{c}}^{R}(\bar{b})+\max \left\{d\left(0_{n}, \mathcal{F}^{o p}(\bar{\pi})\right), \frac{e\left(\mathcal{E}^{o p}(\bar{\pi}), 0_{n}\right)}{\|P\|_{*}}\right\} \\
& \leq \operatorname{lip} \vartheta^{R}(\bar{\pi}) \leq \operatorname{lip} \vartheta_{\bar{c}}^{R}(\bar{b})+e\left(\mathcal{E}^{o p}(\bar{\pi}), 0_{n}\right) .
\end{aligned}
$$

Thus, when $\|P\|_{*}=1$ (for instance if $\operatorname{span}\left\{\bar{a}_{t}, t \in T\right\}=\mathbb{R}^{n}$, or $\mathbb{R}^{n}$ is endowed with the Euclidean norm) we have

$$
\operatorname{lip} \vartheta^{R}(\bar{\pi})=\operatorname{lip} \vartheta_{\bar{c}}^{R}(\bar{b})+\operatorname{lip} \vartheta_{\bar{b}}^{R}(\bar{c})=\operatorname{lip} \vartheta_{\bar{c}}^{R}(\bar{b})+e\left(\mathcal{E}^{o p}(\bar{\pi}), 0_{n}\right)
$$

In the particular case when $\bar{c} \in \operatorname{int} C, e\left(\mathcal{E}^{o p}(\bar{\pi}), 0_{n}\right)=e\left(\mathcal{F}^{o p}(\bar{\pi}), 0_{n}\right)$.

\subsection{On the global Lipschitz modulus}

We start by showing that

$$
\mathrm{g}-\operatorname{lip} \vartheta^{R}=+\infty
$$

so that we have to restrict it somehow in order to get some global information. Indeed, since obviously $\mathrm{g}$-lip $\vartheta^{R} \geq \sup \left(\pi \in \operatorname{dom} \mathcal{F}^{o p} \operatorname{lip} \vartheta^{R}(\pi)\right.$, according to Theorem 5 it is enough to show $\sup _{\pi \in \operatorname{dom} \mathcal{F}^{o p}} e\left(\mathcal{E}^{o p}(\pi), 0_{n}\right)=+\infty$. To do this, consider any basis of $\operatorname{span}\left\{\bar{a}_{t}, t \in T\right\}$, say $\left\{\bar{a}_{t_{1}}, \ldots, \bar{a}_{t_{k}}\right\}$, and any unbounded sequence $\left\{x^{r}\right\}_{r \in \mathbb{N}} \subset$ span $\left\{\bar{a}_{t}, t \in T\right\}$. Define $b_{t}^{r}:=\bar{a}_{t}^{\prime} x^{r}$, for all $t \in T$ and $r \in \mathbb{N}$. Then, clearly $x^{r} \in \mathcal{E}^{o p}\left(0_{n}, b^{r}\right)$ for all $r \in \mathbb{N}$.

In the sequel, fix $\bar{\pi}=(\bar{c}, \bar{b}) \in \operatorname{dom} \mathcal{F}^{o p}$, take any $\rho>0$ and consider $V_{\rho}:=$ $\left\{\pi \in \operatorname{dom} \mathcal{F}^{o p}:\|\pi-\bar{\pi}\|<\rho\right\}$. We are concerned with

$$
\left.\operatorname{g-lip} \vartheta\right|_{V_{\rho}}:=\sup _{\pi, \tilde{\pi} \in V_{\rho}, \pi \neq \tilde{\pi}} \frac{|\vartheta(\pi)-\vartheta(\tilde{\pi})|}{\|\pi-\tilde{\pi}\|} .
$$

Obviously, g-lip $\left.\vartheta\right|_{V_{\rho}} \geq \sup _{\pi \in V_{\rho}} \operatorname{lip} \vartheta^{R}(\pi)$. In order to obtain a point-based upper bound for $\mathrm{g}$-lip $\left.\vartheta\right|_{V_{\rho}}$, we introduce the following notation for a matrix $M \subset \mathbb{R}^{p \times q}$ with rank $q \leq p$ :

$$
\mathcal{I}(M):=\max \left\{\left\|L^{-1}\right\|: L \text { is a rank } q \text { square submatrix of } M\right\},
$$

where $\left\|L^{-1}\right\|:=\max _{\|u\| \leq 1}\left\|L^{-1} u\right\|$ is associated with some given norms in $\mathbb{R}^{p}$ and $\mathbb{R}^{q}$. 
As in the proof of Theorem 5 , set $k:=\operatorname{dim} \operatorname{span}\left\{\bar{a}_{t}, t \in T\right\}$ and let $\left\{v_{k+1}, \ldots, v_{n}\right\}$ be a basis of $\left\{\bar{a}_{t}, t \in T\right\}^{\perp}$ in the case when $k<n$. Let $Q$ denote the matrix whose rows are $v_{k+1}^{\prime}, \ldots, v_{n}^{\prime}$ (in the forthcoming expressions, $Q$ is an 'empty matrix' when $k=n)$. In the next theorem, $A$ denotes the matrix whose rows are $\bar{a}_{t}^{\prime}, t \in T$. Also denote by $I_{m}$ the identity matrix of order $m$ (the cardinality of $T$ ).

Theorem 6 With the notation above,

$$
\operatorname{g}-\left.\operatorname{lip} \vartheta\right|_{V_{\rho}} \leq \mathcal{I}\left(\begin{array}{l}
A \\
Q
\end{array}\right)(\|\bar{b}\|+\rho)+\mathcal{I}\left(\begin{array}{c}
A^{\prime} \\
-I_{m}
\end{array}\right)(\|\bar{c}\|+\rho)
$$

where $\mathcal{I}\left(\begin{array}{l}A \\ Q\end{array}\right)$ is associated with the norms $\|\cdot\|$ in $\mathbb{R}^{n}$ and $\|\cdot\|_{\infty}$ in $\mathbb{R}^{m+k}$ and $\mathcal{I}\left(\begin{array}{c}A^{\prime} \\ -I_{m}\end{array}\right)$ is associated with $\|\cdot\|_{1}$ in $\mathbb{R}^{m}$ and $\|c\|_{*}+\|\lambda\|_{1}$ for $\left(\begin{array}{l}c \\ \lambda\end{array}\right) \in \mathbb{R}^{n+m}$.

Proof Take sequences $\left\{\pi^{r}:=\left(c^{r}, b^{r}\right)\right\}_{r \in \mathbb{N}},\left\{\tilde{\pi}^{r}:=\left(\widetilde{c}^{r}, \widetilde{b}^{r}\right)\right\}_{r \in \mathbb{N}} \subset V_{\rho}$ such that

$$
\mathrm{g}-\left.\operatorname{lip} \vartheta\right|_{V_{\rho}}=\lim _{r \rightarrow \infty} \frac{\left|\vartheta\left(\pi^{r}\right)-\vartheta\left(\tilde{\pi}^{r}\right)\right|}{\left\|\pi^{r}-\tilde{\pi}^{r}\right\|} .
$$

If $b^{r}=\widetilde{b}^{r}$ for all $r$ large enough, one has, from Proposition 4(i),

$$
\begin{aligned}
\left.\operatorname{g-lip} \vartheta\right|_{V_{\rho}} & =\lim _{r \rightarrow \infty} \frac{\left|\vartheta\left(c^{r}, b^{r}\right)-\vartheta\left(\widetilde{c}^{r}, b^{r}\right)\right|}{\left\|c^{r}-\widetilde{c}^{r}\right\|_{*}} \\
& \leq \limsup _{r \rightarrow \infty} \operatorname{g-lip} \vartheta_{b^{r}}^{R} \leq \limsup _{r \rightarrow \infty} e\left(\mathcal{E}\left(b^{r}\right), 0_{n}\right) .
\end{aligned}
$$

For each $r$, any $x^{r} \in \mathcal{E}\left(b^{r}\right)$ may be written as $x^{r}=\left(\begin{array}{c}A_{D} \\ Q\end{array}\right)^{-1}\left(\begin{array}{c}b_{D}^{r} \\ 0_{n-k}\end{array}\right)$, for some rank $k$ submatrix $A_{D}$ of $A$, with rows $\bar{a}_{t}^{\prime}, t \in D$, and the corresponding $b_{D}^{r}=\left(b_{t}^{r}\right)_{t \in D}$. Accordingly,

$$
\left\|x^{r}\right\| \leq\left\|\left(\begin{array}{c}
A_{D} \\
Q
\end{array}\right)^{-1}\right\|\left\|b^{r}\right\|_{\infty} \leq \mathcal{I}\left(\begin{array}{c}
A \\
Q
\end{array}\right)\left(\|\bar{b}\|_{\infty}+\rho\right)
$$

Thus,

$$
\operatorname{g}-\left.\operatorname{lip} \vartheta\right|_{V_{\rho}} \leq \mathcal{I}\left(\begin{array}{l}
A \\
Q
\end{array}\right)\left(\|\bar{b}\|_{\infty}+\rho\right)
$$

The case when $c^{r}=\widetilde{c}^{r}$ for all $r$ large enough, analogously entails, from Proposition 4(ii),

$$
\mathrm{g}-\left.\operatorname{lip} \vartheta\right|_{V_{\rho}} \leq \limsup _{r \rightarrow \infty} \mathrm{g}-\operatorname{lip} \vartheta_{c^{r}}^{R} \leq \limsup _{r \rightarrow \infty} e_{1}\left(\operatorname{extr} \Lambda\left(c^{r}\right), 0_{m}\right) \leq \mathcal{I}\left(\begin{array}{c}
A^{\prime} \\
-I_{m}
\end{array}\right)\left(\|\bar{c}\|_{*}+\rho\right)
$$

In the remaining case, we may assume, by taking suitable subsequences if necessary, that $b^{r} \neq \widetilde{b}^{r}$ and $c^{r} \neq \widetilde{c}^{r}$ for all $r$. Also observe that, according to primal-dual 
feasibility, $\left(c^{r}, \widetilde{b}^{r}\right) \in V_{\rho}$ for all $r$. Then, we have

$$
\begin{aligned}
\left.\operatorname{g-lip} \vartheta\right|_{V_{\rho}} & =\lim _{r \rightarrow \infty} \frac{\left|\vartheta\left(\pi^{r}\right)-\vartheta\left(\widetilde{c}^{r}, b^{r}\right)+\vartheta\left(\widetilde{c}^{r}, b^{r}\right)-\vartheta\left(\tilde{\pi}^{r}\right)\right|}{\left\|\pi^{r}-\widetilde{\pi}^{r}\right\|} \\
& \leq \limsup _{r \rightarrow \infty} \frac{\left|\vartheta\left(\pi^{r}\right)-\vartheta\left(\widetilde{c}^{r}, b^{r}\right)\right|}{\left\|c^{r}-\widetilde{c}^{r}\right\|_{*}}+\limsup _{r \rightarrow \infty} \frac{\left|\vartheta\left(\widetilde{c}^{r}, b^{r}\right)-\vartheta\left(\tilde{\pi}^{r}\right)\right|}{\left\|b^{r}-\widetilde{b}^{r}\right\|_{\infty}} \\
& \leq \mathcal{I}\left(\begin{array}{c}
A \\
Q
\end{array}\right)\left(\|\bar{b}\|_{\infty}+\rho\right)+\mathcal{I}\left(\begin{array}{c}
A^{\prime} \\
-I_{m}
\end{array}\right)\left(\|\bar{c}\|_{*}+\rho\right) .
\end{aligned}
$$

\section{Conclusions}

This paper is primarily concerned with lower estimations of the Lipschitz modulus of the optimal value of linear programs in two perturbations settings: objective function (tilt) perturbations and canonical perturbations (tilt ones together with right-hand side-RHS - perturbations). In both cases, we can find in the literature upper bounds (Theorems 1 and 2), whereas only for RHS perturbations the exact modulus is known (see Remark 3).

The new point-based lower bounds given in this paper have been obtained by using a projection-based approach. Specifically, we have appealed to $\|P\|_{*}$, which denotes the dual norm of the orthogonal projection on the linear subspace spanned by the lefthand side coefficients of the system with respect to the usual inner product in $\mathbb{R}^{n}$. It is known that this value is always greater than or equal to 1, and, in particular, equals 1 when either the feasible set contains no lines or $\mathbb{R}^{n}$ is endowed with the Euclidean norm.

On tilt perturbations, Theorem 3 establishes a new lower bound which, combined with the antecedent Theorem 1, completes the picture for this framework (see Corollary 2). In this sense, Example 4 shows that this new lower bound may be less or greater than the previously known one, while Example 3 shows that both the new lower bound and the known upper bound may be attained. In the cases when $\|P\|_{*}$ equals 1 (previously mentioned), we obtain the exact value of the Lipschitz modulus which coincides with the previously known upper bound. As for canonical perturbations, a lower bound on the corresponding Lipschitz modulus is given in Theorem 5, which, together with Theorem 2, completes the study. Additionally, when $\|P\|_{*}$ equals 1 , the Lipschitz modulus under canonical perturbations can be written as the sum of the corresponding moduli under tilt and RHS perturbations (see Corollary 3).

Finally, we have also studied the global Lipschitz modulus in the same perturbation settings. As shown in Theorem 4, the corresponding modulus under tilt perturbations can be estimated through lower and upper bounds, while the exact modulus arises under RHS perturbations. In both cases, the expressions for the global case are the analogous to the local ones. Since the global Lipschitz modulus under canonical perturbations turns out to be always infinite, we restrict our approach to problems in a fixed ball, centered at the nominal problem and with a fixed radius, in order to obtain a finite upper bound (see Section 5.2). 
Acknowledgements The authors wish to thank the anonymous referees for their valuable critical comments.

Funding Open Access funding provided thanks to the CRUE-CSIC agreement with Springer Nature.

Open Access This article is licensed under a Creative Commons Attribution 4.0 International License, which permits use, sharing, adaptation, distribution and reproduction in any medium or format, as long as you give appropriate credit to the original author(s) and the source, provide a link to the Creative Commons licence, and indicate if changes were made. The images or other third party material in this article are included in the article's Creative Commons licence, unless indicated otherwise in a credit line to the material. If material is not included in the article's Creative Commons licence and your intended use is not permitted by statutory regulation or exceeds the permitted use, you will need to obtain permission directly from the copyright holder. To view a copy of this licence, visit http://creativecommons.org/licenses/by/4.0/.

\section{References}

1. Bank, B., Guddat, J., Klatte, D., Kummer, B., Tammer, K.: Non-Linear Parametric Optimization. Akademie-Verlag, Berlin 1982, and Birkhäuser, Basel (1983)

2. Bertsimas, D., Tsitsiklis, J.N.: Introduction to Linear Optimization. Athena Scientific, Nashua (1997)

3. Dontchev, A.L., Rockafellar, R.T.: Implicit Functions and Solution Mappings: A View from Variational Analysis. Springer, New York (2009)

4. Gisbert, M.J., Cánovas, M.J., Parra, J., Toledo, F.J.: Calmness of the optimal value in linear programming. SIAM J. Optim. 28, 2201-2221 (2018)

5. Gisbert, M.J., Cánovas, M.J., Parra, J., Toledo, F.J.: Lipschitz modulus of the optimal value in linear programming. J. Optim. Theory Appl. 182, 133-152 (2019)

6. Goberna, M.A., López, M.A.: Linear Semi-Infinite Optimization. Wiley, Chichester (UK) (1998)

7. Kanzow, C., Qi, H., Qi, L.: On the Minimum Norm Solution of Linear Programs. J. Optim. Theory Appl. 116, 333-345 (2003)

8. Klatte, D., Kummer, B.: Nonsmooth Equations in Optimization: Regularity, Calculus, Methods and Applications, Nonconvex Optim. Appl. 60, Kluwer Academic, Dordrecht, The Netherlands (2002)

9. Mordukhovich, B.S.: Variational Analysis and Generalized Differentiation, I: Basic Theory. Springer, Berlin (2006)

10. Nozicka, F., Guddat, J., Hollatz, H., Bank, B.: Theorie der Linearen Parametrischen Optimierung. Akademie-Verlag, Berlin (1974)

11. Renegar, J.: Some perturbation theory for linear programming. Math. Program. 65, 73-91 (1994)

12. Robinson, S.M.: Some continuity properties of polyhedral multifunctions. Math. Progr. Study 14, 206-214 (1981)

13. Rockafellar, R.T.: Convex Analysis. Princeton University Press, Princeton (1970)

14. Rockafellar, R.T., Wets, R.J.-B.: Variational Analysis. Springer, Berlin (1998)

15. Tikhonov, A.N., Arsenin, V.Y.: Solutions of Ill-Posed Problems. Halsted Press, Wiley, New York (1977)

16. Walkup, D.W., Wets, R.J.-B.: Lifting projections of convex polyhedra. Pacific J. Math. 28, 465-475 (1969)

17. Wets, R.J.-B.: On the continuity of the value of a linear program and of related polyhedral-valued multifunctions. Math. Progr. Study 24, 14-29 (1985)

Publisher's Note Springer Nature remains neutral with regard to jurisdictional claims in published maps and institutional affiliations. 